\title{
Nanoparticle Certified Reference Materials: Development and Microscopical Characterization
}

\author{
Michelle R. Cavaliere
}

MVA Scientific Consultants, 3300 Breckinridge Blvd. Suite 400, Duluth, GA 30096

Nanotechnology is evolving rapidly with a pivotal role in many industrial sectors and a high economic impact [1]. Estimates predict that by next year, \$2.6 trillion in manufactured goods will incorporate nanotechnology, which is approximately $15 \%$ of total global output [2]. A marker of this consistent growth is the number of consumer products containing nanomaterials recorded in the Project on Emerging Nanotechnologies' database, which predicts the number of products to reach 3,400 by the year 2020 [3]. With this surge in nanotechnology, the need arises for certified reference materials (CRM) specifically designed to meet the needs of nanomanufacturing. Development of a CRM is a lengthy process requiring multiple steps to ensure material suitability, homogeneity, stability, accuracy and precision with respect to the property being certified (i.e. size). Recently, we have participated in the certification campaign for two such nanoparticle CRM issued by the European Commission Joint Research Centre Institute for Reference Materials and Measurements (IRMM).

Our role in the CRM certification campaign was a multistage process utilizing transmission electron microscopy (TEM) to examine particle size and morphology. First, we were employed as an independent laboratory to evaluate the raw materials from which the CRM would be prepared (Figure 1). Examination of the two candidates revealed the starting material for the larger diameter material to be somewhat more polydisperse compared to the smaller diameter material. Once the colloidal silica materials were deemed suitable CRM candidates, we participated in an interlaboratory study using a colloidal silica proficiency test material to demonstrate technical competence and proficiency in the sizing of nanoparticles. A final characterization study was performed with comparison of results from multiple expert laboratories in order to provide a certified spherical diameter specific to the sizing technique used (eg. DLS, CLS, SEM, TEM). A TEM imaging protocol over multiple days, including tests on a Quality Control Material, was followed by particle measurements using NIH ImageJ software [4] with a minimum of 500 particles measured. Figures 2 and 3 show TEM results obtained at MVA Scientific Consultants as compared to the other participants $[5,6]$.

Ultimately, the results led to the first certified silica nanoparticle reference materials available worldwide. The colloidal silica CRM provide a means to check the performance of instruments or methods used to characterize the size distribution of nanoparticles in three different capacities: 1) comparison of an analytical result with a certified value, 2) use in quality control charts, and 3) use as a calibrant [6].

\section{References}

[1] "Nanotechnology Market Forecast to 2014", RNCOS Industry Research Solutions, October 2012.

[2] "The Nanotech Report", 5 ${ }^{\text {th }}$ Ed., Lux Research, New York, 2007.

[3] The Project On Emerging Nanotechnologies News Archive. "Nanotech-enabled Consumer Products Continue to Rise" http://www.nanotechproject.org/news/archive/9231/ (accessed February 2013).

[4] Rasband, W.S., ImageJ, U.S. National Institutes of Health, Bethesda, Maryland, USA, http://imagej.nih.gov/ij/, 1997-2012. 
[5] "Certification of Equivalent Spherical Diameters of Silica Nanoparticles in Water Certified Reference Material ERM ${ }^{\mathbb{B}}$-FD100”, European Commission Joint Research Centre Institute for Reference Materials and Measurements, Publications Office of the European Union, Luxembourg 2011. [6] "Certification of the Equivalent Spherical Diameters of Silica Nanoparticles in Aqueous Solution Certified Reference Material ERM ${ }^{\mathbb{B}}$-FD304”, European Commission Joint Research Centre Institute for Reference Materials and Measurements, Publications Office of the European Union, Luxembourg 2012.
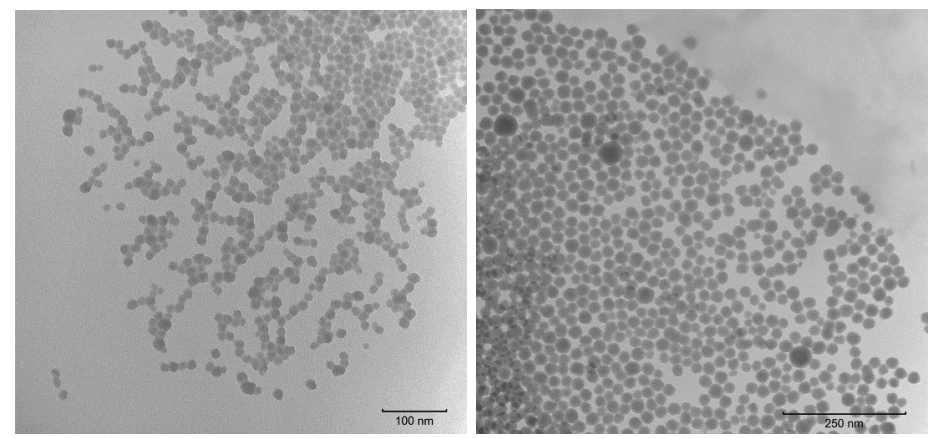

Figure 1. TEM images of starting materials used for preparation of ERM-FD100 (left) and ERMFD304 (right) certified reference materials.
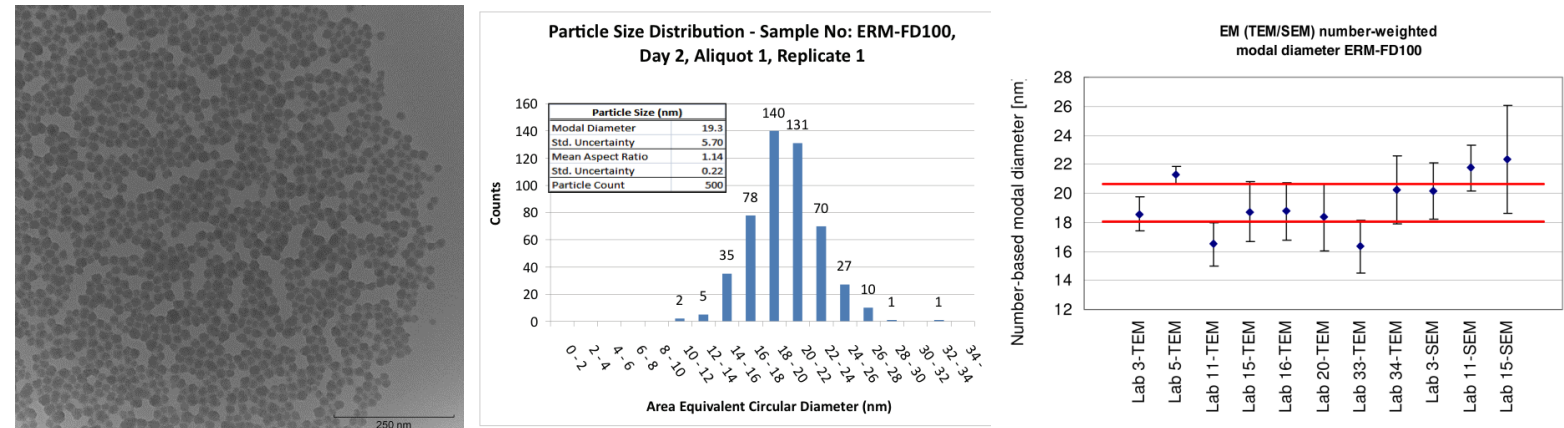

Figure 2. Representative TEM image taken during certification study of ERM-FD100 (left), modal diameter distribution of ERM-FD100 from size measurements in our laboratory of aliquot 1, replicate 1, day 2 (center), and number weighted modal diameter of ERM-FD100 results from all participating laboratories [5] (right).
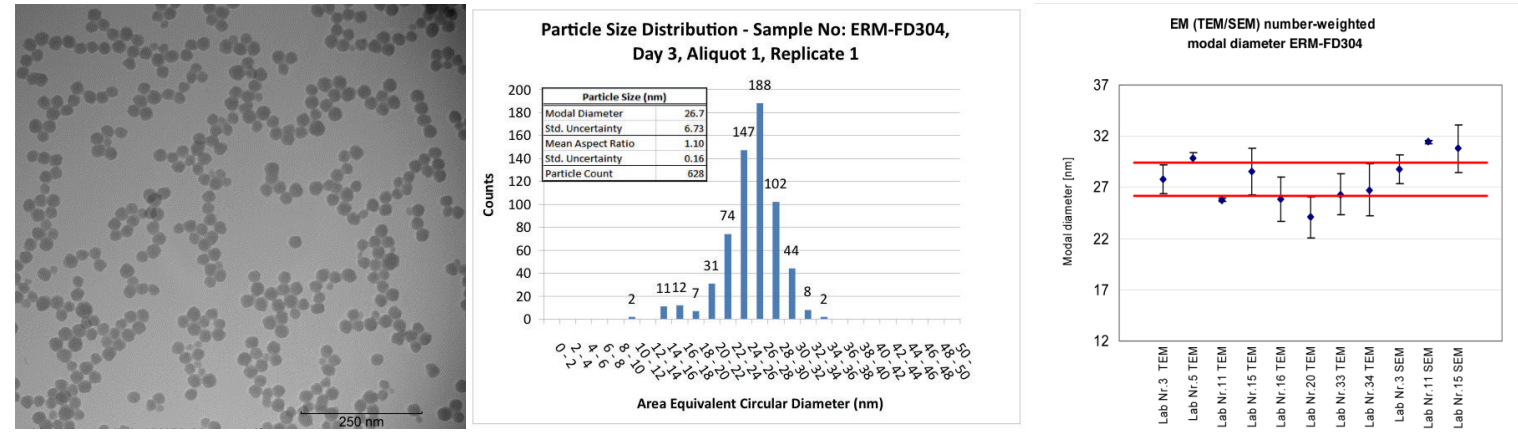

Figure 3. Representative TEM image taken during certification study of ERM-FD304 (left), modal diameter distribution of ERM-FD304 from size measurements in our laboratory of aliquot 1, replicate 1, day 3 (center), and number weighted modal diameter of ERM-FD304 results from all participating laboratories [6] (right). 